\title{
An Adjusted Gaussian Skin-Color Model Based on Principal Component Analysis
}

\author{
Zhi-Gang Fan and Bao-Liang Lu \\ Department of Computer Science and Engineering, Shanghai Jiao Tong University, \\ 1954 Hua Shan Road, Shanghai 200030, China \\ zgfan@sjtu.edu.cn, blu@cs.sjtu.edu.cn
}

\begin{abstract}
By combining the two standard paradigms of unsupervised learning, Principal Component Analysis (PCA) and Gaussian density estimation, this paper proposes an adjusted Gaussian skin-color model for skin-color detection. This method is more robust than the standard Gaussian model because it can weaken the bias caused by noise and enhance the fitness of the mathematical model. The experiments show that this method works well for the real-world images with complex backgrounds.
\end{abstract}

\section{Introduction}

Automatic detection of human faces is a very difficult task. Face detection in color images that begin with skin color modeling has been the topic of extensive research for the several past decades. In recent years, surveys on face detection have been made [8], 9], [4]. Some adaptive and unsupervised methods for skincolor modeling have been introduced in resent years [7], 3], 1]. We propose an adjusted Gaussian skin-color model based on Principal Component Analysis (PCA) for skin-color detection. We use single Gaussian model and don't use Gaussian mixture models because we find that, in our dataset, the distribution of skin-color data is unimodal. Some researchers have the same point of view [7. We show that our model significantly outperforms the standard Gaussian model.

\section{Adjusted Gaussian Skin-Color Model}

\subsection{Gaussian Model and PCA}

The multivariate Gaussian density is a very important and unique density function in statistical theory. It may be viewed as the central idea in second-order statistics. The distribution of an n-dimensional random vector $x$ is Gaussian if its probability density function has the form:

$$
p_{x}(x)=\frac{1}{(2 \pi)^{n / 2}\left|C_{x}\right|^{1 / 2}} \exp \left(-\frac{1}{2}\left(x-m_{x}\right)^{T} C_{x}^{-1}\left(x-m_{x}\right)\right)
$$

where $m_{x}$ is its mean, and $C_{x}$ is the covariance matrix of $x$. 
Principal component analysis (PCA) is a popular method for features extraction and pattern representation. It can find the projection directions that maximize the total scatter across all variables and remove the mutual correlation between the elements. Denote the training set of $m$ samples by $X=$ $\left(x_{1}, x_{2}, \ldots, x_{m}\right) \subset R^{n \times m}$, where $x$ is an $n$-dimensional vector. Define the covariance matrix as follows:

$$
C_{x}=\frac{1}{m} \sum_{i=1}^{m}\left(x_{i}-m_{x}\right)\left(x_{i}-m_{x}\right)^{T}
$$

Then, the eigenvalues and eigenvectors of the covariance $C_{x}$ are calculated. Let $A=\left(a_{1}, a_{2}, \ldots, a_{r}\right) \subset R^{n \times r}(r \leq n)$ be the $r$ eigenvectors corresponding to the $r$ largest eigenvalues $\left\{\lambda_{1}, \lambda_{2}, \ldots, \lambda_{r}\right\}$. Thus, for the original random vector $x$ which belongs to set $X=\left(x_{1}, x_{2}, \ldots, x_{m}\right) \subset R^{n \times m}$, its corresponding eigenfeature $y$, belonging to the set $Y=\left(y_{1}, y_{2}, \ldots, y_{m}\right) \subset R^{r \times m}$, can be obtained by projecting $x$ into the eigenfeature space as follows:

$$
y=A^{T}\left(x-m_{x}\right)
$$

The transformed vector $y$ is a random vector with zero mean and its covariance matrix $D$ is related to that of $x$ by the following equation:

$$
D=A^{T} C_{x} A
$$

where $D$ is a diagonal matrix having the eigenvalues of $C_{x}$ along its diagonal. According to equation (4), we can obtain the following equation:

$$
C_{x}=\left(A^{T}\right)^{-1} D A^{-1}
$$

Then, we can obtain the following equation:

$$
C_{x}^{-1}=A D^{-1} A^{T}
$$

From equations (3) and (6), we have

$$
\begin{aligned}
\left(x-m_{x}\right)^{T} C_{x}^{-1}\left(x-m_{x}\right) & =\left(x-m_{x}\right)^{T}\left(A D^{-1} A^{T}\right)\left(x-m_{x}\right) \\
& =\left(\left(x-m_{x}\right)^{T} A\right) D^{-1}\left(A^{T}\left(x-m_{x}\right)\right) \\
& =y^{T} D^{-1} y \\
& =\sum_{i=1}^{r} \frac{y_{i}^{2}}{\lambda_{i}}
\end{aligned}
$$

By using the right part of equation (7), we can change equation (11) into the following form:

$$
p_{x}(x)=\frac{1}{(2 \pi)^{n / 2} \prod_{i=1}^{r} \lambda_{i}^{1 / 2}} \exp \left(-\frac{1}{2} \sum_{i=1}^{r} \frac{y_{i}^{2}}{\lambda_{i}}\right)
$$




\subsection{Adjusted Model}

Through PCA, the Gaussian density function can be changed into the form as equation (8). When $r=n$, the equation (8) is equal to the original one, equation (11), but it can transform the coordinate axes into the principal axes of Gaussian model. Thus, not only we can calculate more feasible, but also, the more important, we can easily evaluate the fitness of the Gaussian model along the principal axes directions. In practice, the Gaussian skin-color model always has considerable bias because of the noise in the sample set. Fig 1 shows such situation, in which a histogram of the skin-color samples projected on the second principal axes of the Gaussian skin-color model is illustrated. We denote the histogram as $f(x)$. The mean is located on the zero point. The bias can obviously be seen because the main peak of $f(x)$ floats away from the mean. The reason for the bias is that the noise is inevitably mixed into the sample

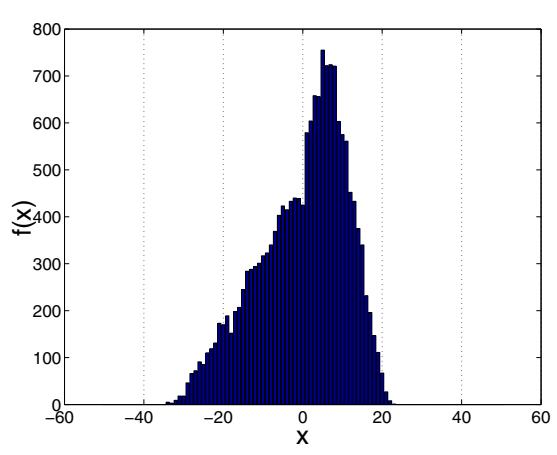

Fig. 1. The histogram $f(x)$

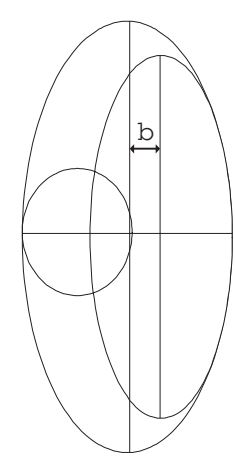

Fig. 2. The bias of the Gaussian model affected by noise

set when sampling. Usually, the Gaussian model is disturbed by the noise on one side such as the situation shown in Fig 2, in which the two ellipses denote two Gaussian models, the small circle denotes the noise, and the parameter $b$ denotes the bias. The small ellipse is disturbed by the circle on the left side, so, it is replaced by the big ellipse which produces the bias $b$. To adjust the bias, we must move the vertical principal axes to right.

Because of the big bias, we must adjust the model to weaken the bias and enhance the fitness. According to equation (8), we can effectively adjust the Gaussian model along the principal axes direction. We can adjust different principal axes independently at the same time disregarding relationship among them because these axes are uncorrelated. On the $i$ th principal axes, we define the bias as two components, the bias on mean as $b_{i}$ and the bias on variance as $c_{i}$. Thus, we can define the adjusted Gaussian skin-color model in the form:

$$
p_{x}(x)=\frac{1}{(2 \pi)^{n / 2} \prod_{i=1}^{r}\left(c_{i} \lambda_{i}\right)^{1 / 2}} \exp \left(-\frac{1}{2} \sum_{i=1}^{r} \frac{\left(y_{i}-b_{i}\right)^{2}}{c_{i} \lambda_{i}}\right)
$$




\subsection{Measurement of the Biases $b_{i}$ and $c_{i}$}

On the $i$ th principal axes, we can measure the biases $b_{i}$ and $c_{i}$ according to the histogram of the training samples projected on this principal axes. The histogram $f(x)$ shown in Fig. 1 is just this type of histogram. Obviously, the function $f(x)$ is a noisy function and should be smoothed. We use a low-pass filter $g(x)$ to smooth $f(x)$ through convolution operation:

$$
h(x)=f(x) * g(x)
$$

Assume function $h(x)$ reaches its maximum value at point $x_{m}: \max (f(x))=$ $f\left(x_{m}\right)$. Specifically, we begin the convolution operation from the point $x_{m}$, and go on along the two sides of this point respectively. The smoothed histogram $h(x)$ is shown in Fig 3. In order to measure the biases, we have to locate the

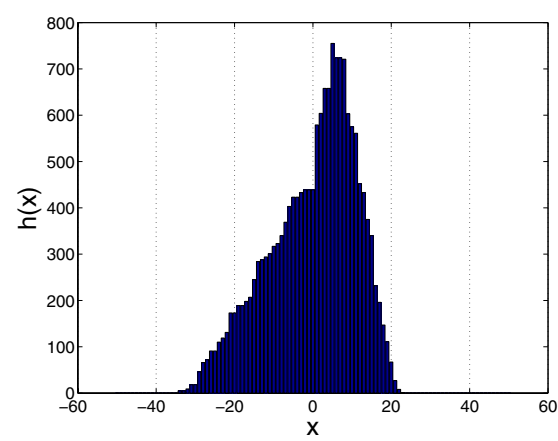

Fig. 3. The smoothed histogram $h(x)$

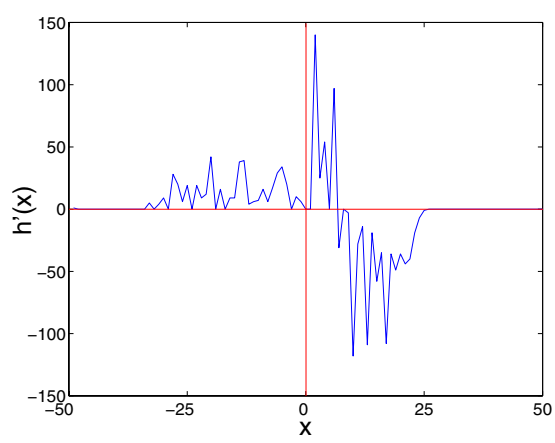

Fig. 4. The $h^{\prime}(x)$

main peak of the histogram. Differentiating the function $h(x)$ we can obtain its derivative $h^{\prime}(x)$ and produce reliable peaks at the inflection points. Fig 4 shows $h^{\prime}(x)$. The two mutually perpendicular lines indicate the location of zero point. We can obviously see the high peak part is on the right side of the mean point. Assume that the high peak part is in the interval $\left(x_{1}, x_{2}\right)$. For this task, selecting the interval $\left(x_{1}, x_{2}\right)$, careful consideration should be made. In practice, the interval $\left(x_{1}, x_{2}\right)$ should include the point $x_{m}$ and cannot include the deep valleys parts whose bottoms reach the zero line and two sides are high peaks.

High peak part of $h^{\prime}(x)$ in the interval $\left(x_{1}, x_{2}\right)$ indicates the main peak of $h(x)$. Thus, to adjust the biases, we should move mean point to the central point of the interval $\left(x_{1}, x_{2}\right)$. Assume $h(x)>0$ in the interval $\left(x_{n}, x_{p}\right)$. On the $i$ th principal axes, we can measure the biases through the following formulas :

$$
\begin{aligned}
d & =\left(x_{2}-x_{1}\right) / 2 \\
d_{1} & =x_{p}-x_{n} \\
b_{i} & =d+x_{1} \\
c_{i} & =2 d / d_{1}
\end{aligned}
$$


However, in practice, on some principal axes the biases are too small to be adjusted necessarily. Thus, on the $i$ th principal axes, we change the formulas (13), and (14) to the following forms:

$$
\begin{aligned}
& b_{i}= \begin{cases}d+x_{1} & \left|d+x_{1}\right|>Q_{i} \\
0 & \text { otherwise }\end{cases} \\
& c_{i}= \begin{cases}2 d / d_{1} & \left|d+x_{1}\right|>Q_{i} \\
1 & \text { otherwise }\end{cases}
\end{aligned}
$$

where $Q_{i}$ is a user defined constant threshold on the $i$ th principal axes.

\section{Implementation and Experiments}

We use the standard Gaussian skin-color model and adjusted Gaussian skin-color model respectively in our experiments for comparison study. Fig 5 shows the

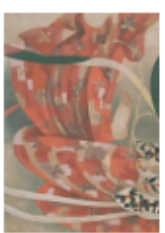

(a)

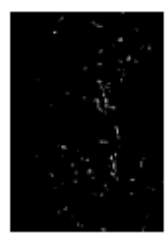

(b)

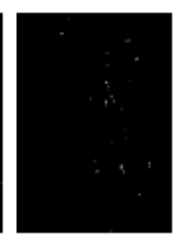

(c)

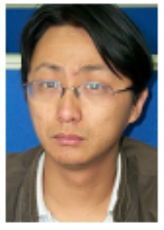

(a)

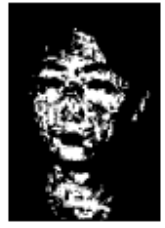

(b)

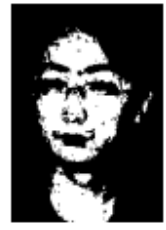

(c)
Fig. 5. (a) A non-face image; (b) Binary image after segmentation based on standard Gaussian model; (c) Binary image after segmentation based on adjusted Gaussian model
Fig. 6. (a) A face image; (b) Binary image after segmentation based on standard Gaussian model; (c) Binary image after segmentation based on adjusted Gaussian model

detection result on non-face images. The segmentation (Fig 5 (b)) based on standard Gaussian model shows that much non-skin color is misjudged as skin color. In contrast, the misjudgment rate is very low in the segmentation (Fig.5 (c)) by using our adjusted Gaussian model. Fig 6 shows the detection result on face images. The segmentation (Fig[6(c) ) obtained through adjusted Gaussian model is much better than the segmentation (Fig 6(b) ) processed by using standard Gaussian model.

Table 1 shows the number of skin-color pixels detected by adjusted model is much larger than that detected by the standard model. Through Table 1, we can see that the adjusted Gaussian skin-color model outperforms the standard Gaussian skin-color model. 
Table 1. Result of experiments on face images

\begin{tabular}{c|c|c|c}
\hline \hline Methods & $\begin{array}{c}\text { No. all pixels } \\
\text { in test images }\end{array}$ & $\begin{array}{c}\text { No. the pixels } \\
\text { be detected }\end{array}$ & $\begin{array}{c}\text { Ratio between detected pixels } \\
\text { and all pixels (\%) }\end{array}$ \\
\hline Standard model & 2665872 & 425007 & $15.94 \%$ \\
\hline Adjusted model & 2665872 & 669042 & $25.10 \%$ \\
\hline \hline
\end{tabular}

\section{Conclusions}

As an improvement to standard Gaussian skin-color model, we analyze the adjusted Gaussian skin-color model based on PCA. The experimental results indicate that in face images, our model can detect much more skin-color than the standard model; while in non-face images, very lower misjudgment rate is obtained with our model. Furthermore, the flexibility of our adjusted model can be used in many related color-detection system and applied to real-time application.

Acknowledgements. The authors thank Bin Huang for his help and discussion during preparing this paper. This work was partially supported by the National Natural Science Foundation of China via the grant NSFC 60375022.

\section{References}

1. Bergasa, L. M., Mazo, M., Gardel, A., Sotelo, M. A., Boquete, L.: Unsupervised and Adaptive Gaussian Skin-Color Model, Image and Vision Computing, vol. 18, (2000) 987-1003

2. Greenspan, H., Goldberger, J., Eshet, I.: Mixture Model for Face Color Modeling and Segmentation, Pattern Recognition Letters, Vol. 22, (2001) 1525-1536

3. Soriano, M., Martinkauppi, B., Huovinenb, S., Laaksonenc, M.: Adaptive skin color modeling using the skin locus for selecting training pixels, Pattern Recognition, Vol. $36,(2003)$ 681-690

4. Vezhnevets, V., Sazonov, V., Andreeva, A.: A Survey on Pixel-Based Skin Color Detection Techniques, In Proc. Graphicon-2003, Moscow, Russia (2003) 85-92

5. Moghaddam, B., Pentland, A.: Probabilistic visual learning for object representation, IEEE Trans. Pattern Anal. and Machine Intell. , vol. 19, no. 7, (1997) 696-710

6. Turk, M., Pentland, A.: Eigenfaces for Recognition, Journal of Cognitive Neuroscience, vol. 3, no. 1, (1991) 71-86

7. Hsu, R. L., Mottaleb, M. A., Jain, A. K.: Face detection in color images, IEEE Trans. Pattern Anal. and Machine Intell. , vol. 24, no. 5, (2002) 696-706

8. Yang, M. H., Kriegman, D., Ahuja, N.: Detecting Faces in Images: A Survey, IEEE Trans. Pattern Anal. and Machine Intell. , vol. 24, no. 1, (2002) 34-58

9. Hjelm, E., Low, B. K.: Face Detection: A Survey, Computer Vision and Image Understanding, vol. 83, no. 3, (2001) 236-274 